\title{
Comparison of Radiological Criteria (RECIST - MASS - SACT -Choi) in Antiangiogenic Therapy of Renal Cell Carcinoma
}

\author{
Vancini C. ${ }^{1, *}$, De Falco Alfano D. ${ }^{2}$, Abousiam R.N. ${ }^{2}$, Totaro M. ${ }^{2}$, Murri Dello Diago N. ${ }^{3}$, Giganti M. ${ }^{2}$ \\ ${ }^{1}$ Department of Radiology, AUSL Romagna, Bufalini Hospital, Italy \\ ${ }^{2}$ Department of Morphology, Surgery and Experimental Medicine, Diagnostic Imaging Section, University of Ferrara, Italy \\ ${ }^{3}$ Department of Radiology, SS. Annunziata Hospital, Italy
}

Copyright $\bigcirc 2016$ by authors, all rights reserved. Authors agree that this article remains permanently open access under the terms of the Creative Commons Attribution License 4.0 international License

\begin{abstract}
Aim: To compare the diagnostic accuracy of different radiological criteria used in the assessments of antiangiogenic response in patients with metastatic Renal Cell Carcinoma (RCC). Methods: 44 patients were retrospectively evaluated from January 2007 through September 2014, the patients had histologic diagnosis of RCC, existence of target lesions, in therapy with Sorafenib or Sunitinib assumed continuously for 4 weeks for a total of 6 cycles and went through multiphase CT before and 3 months after the therapy. Results: Out of the 44 patients, 21 had Progression Free Survival (PFS) $>250$ days. In this group of patients, all the radiological criteria had high predictive value with slightly higher sensitivity for the RECIST 1.1 criteria (sensitivity $100 \%$ ). In the remaining group of 23 patients (with PFS $<250$ days), RECIST criteria also showed slightly higher specificity values by identifying 9 patients (60.86\%) compared with Choi, MASS and SACT who identified respectively $56.52 \%, 52.17 \%$ and $56.52 \%$ of the patients as having stable disease or indeterminate response to the therapy. Conclusion: All studied radiological criteria had high predictive value with slightly higher sensitivity results for the RECIST 1.1 criteria.
\end{abstract}

Keywords Renal Cell Carcinoma, Assessments of Antiangiogenic Response, Radiological Criteria, RECIST MASS - SACT - Choi

\section{Introduction}

The renal clear cell carcinoma (RCC) represents the $80-85 \%$ of kidney tumors and it is the highest form of carcinoma malignancy in all of the urological tumors. In Europe the RCC represents 3-4\% of all adult cancers with approximately 40,000 new cases a year, a peak of incidence between 60-70 years old and a male-female ratio of 2:1 ${ }^{[1][2][3]}$. The increase of incidence and the improvement of the 5 years survival that occurred in recent years is partly attributable to the progress of diagnostic imaging which made it possible to identify these tumors in an earlier stage, increasingly as occasional findings. In $25-40 \%$ of cases the RCC remain asymptomatic and being detected incidentally when non-invasive imaging is used to investigate a variety of nonspecific symptoms and other abdominal diseases. The most common manifestations of the RCC are hematuria (50-60\% of patients), abdominal pain (40\%) and palpable mass $(30-40 \%)^{[4][5]}$. These symptoms, that were once regarded as the "classic triad" of presentation, in fact are very rare, occur in less than $10 \%$ of patients and are indicative of advanced disease ${ }^{[6]}$. Only $55 \%$ of patients at the time of diagnosis have a tumor confined only in the kidney, while $25-30 \%$ has distant metastasis and $19 \%$ has locally advanced disease. Frequent sites of metastasis are the pulmonary parenchyma $(50-60 \%$ of cases), bones $(30-40 \%)$ and liver $(30-40 \%)^{[4]}$.

\section{Rationale and Purpose}

Since the year 2000 , to evaluate the therapeutic response in patients treated with antiangiogenic therapy, RECIST ${ }^{[7]}$ criteria has been used; these criteria rely on size variations of target lesion and the appearance of new metastases. However, in the following years, several studies have shown the inadequacy of those criteria and therefore new radiological criteria were proposed; the new criteria were no longer one-dimensional but functional criteria that evaluate the attenuation and marked central necrosis of the target lesion. Through those studies, it has been demonstrate that the objective analysis of tumor size and the attenuation on CECT improves the response evaluation $\left(\mathrm{CHOI}{ }^{[8,9]}\right.$, MASS: mass, attenuation, size and structure ${ }^{[10]}$, SACT: size and 
attenuation $\mathrm{CT}^{[11]}$ ). The imaging response categories used by RECIST and CHOI were complete response, partial response, stable disease and progression disease (table 1,2). The imaging response categories used by MASS and SACT criteria were favorable response, indeterminate response or unfavorable response (table 3,4).

The purpose of our retrospective study was to compare the accuracy of different criteria for assessing the response of patients with metastatic RCC treated with antiangiogenic therapy.

Table 1. RECIST 1.1 criteria

\begin{tabular}{|c|c|}
\hline Objective response & RECIST criteria description \\
\hline Complete Response (CR) & $\begin{array}{c}\text { 1. Disappearance of all lesions } \\
\text { 2. No new lesions }\end{array}$ \\
\hline Partial Response (PR) & $\begin{array}{c}\text { 1. Decrease in the sum of target lesions } \\
\text { diameter }>=20 \%\end{array}$ \\
\hline Stable disease (SD) & No criteria for CR, PR, PD \\
\hline Progression disease (PD) & $\begin{array}{c}\text { 1. Increased in tumor size of }>=30 \% ; \\
\text { 2. New lesions }\end{array}$ \\
\hline
\end{tabular}

Table 2. CHOI criteria

\begin{tabular}{|c|c|}
\hline Objective response & CHOI criteria description \\
\hline $\begin{array}{c}\text { Complete } \\
\text { Response (CR) }\end{array}$ & 1. Disappearance of all lesions \\
2. No new lesions
\end{tabular}

Table 3. SACT criteria

\begin{tabular}{|c|l|}
\hline Objective response & \multicolumn{1}{|c|}{ SACT criteria description } \\
\hline $\begin{array}{c}\text { Favorable } \\
\text { response }\end{array}$ & $\begin{array}{l}\text { No new lesions: 1. Decrease in size }>=20 \% ; \\
\text { 2. One or more enhancing lesions with marked } \\
\text { central necrosis or decreased attenuation }> \\
40 \%\end{array}$ \\
\hline $\begin{array}{c}\text { Indeterminate } \\
\text { response }\end{array}$ & $\begin{array}{l}\text { No criteria for favorable or unfavorable } \\
\text { response }\end{array}$ \\
\hline $\begin{array}{c}\text { Unfavorable } \\
\text { response }\end{array}$ & $\begin{array}{l}\text { 1. Increased in size }>=20 \% \text { without marked } \\
\text { central necrosis or decreased attenuation; 2. } \\
\text { New metastases; 3. Marked central fill-in; 4. } \\
\text { New enhancement }\end{array}$ \\
\hline
\end{tabular}

Table 4. MACT criteria

\begin{tabular}{|c|l|}
\hline $\begin{array}{c}\text { Objective } \\
\text { response }\end{array}$ & \multicolumn{1}{|c|}{ MASS criteria description } \\
\hline $\begin{array}{c}\text { Favorable } \\
\text { response }\end{array}$ & $\begin{array}{l}\text { 1. Decrease in size }>=20 \% \text { or in mean } \\
\text { attenuation }>40 \mathrm{UH} \text { at least in one non-lung } \\
\text { target lesion; 2. Decrease in size }>=10 \% \text { and in } \\
\text { mean attenuation }>\text { UH in half of the } \\
\text { non-lung target lesions }\end{array}$ \\
\hline $\begin{array}{c}\text { Indeterminate } \\
\text { Response }\end{array}$ & $\begin{array}{l}\text { No criteria for favorable or unfavorable } \\
\text { response }\end{array}$ \\
\hline $\begin{array}{c}\text { Unfavorable } \\
\text { response }\end{array}$ & $\begin{array}{l}\text { 1. Increased in size }>=20 \% ; 2 . \text { New lesions; 3. } \\
\text { New enhancement; 4. Change from central } \\
\text { necrosis to near complete enhancement of solid } \\
\text { portions }\end{array}$ \\
\hline
\end{tabular}

\section{Materials and Methods}

\subsection{Patient Characteristics}

CT images of 108 patients with metastatic RCC were retrospectively evaluated from January 2007 through December 2012 at the Sant'Anna University Hospital of Ferrara. Of those patients, 44 met all the following inclusion criteria and therefore selected:

- Certain diagnosis of RCC.

- First line therapy with Sorafenib or Sunitinib.

- Assume the therapy continuously for 4 weeks and for a total of 6 cycles.

- Images acquired with multiphase CT (without contrast medium, in arterial phase, in portal-venous phase) before and after 3 months of therapy.

- Existence of target lesions.

Therefore, the following patients were excluded from the study:

- 34 patients since they were not on the first cycle of treatment.

- 6 patients who suspended the therapy as a result of high toxicity.

- 5 patients who had not taken the therapy for more than a week for various reasons.

- 9 patients since they did not had the arterial phase CT.

- 6 patients that had not performed a post-therapy CT scan after 3 months.

- 4 patients in whom no target lesions were found.

The remaining 44 patients had the following characteristics:

- $\quad 39$ males and 5 females.

- Age range between 50 and 80 years.

- 38 in therapy with the tyrosine kinase inhibitor Sorafenib and 6 with Sunitinib.

\section{Preoperative Data Collection}

All CT examinations were acquired at our Institute with 64-layers multi-detector CT. Each CT examination was performed with the CECT method (Contrast-Enhanced CT) of evaluating prior to the initiation of the therapy and after his suspension, additionally in a multiphase mode with an acquisition without contrast medium, in arterial phase and portal phase. The measurement of the lesions occurred always on the axial plane at the stage where the lesion was more evident. An iodate contrast medium was used with a concentration of $1.2 / 1.5 \mathrm{ml} / \mathrm{kg}-350 \mathrm{mg} \mathrm{I} / \mathrm{ml}$. For each patient the post-antiangiogenic therapy $\mathrm{CT}$ images were compared with the pre-therapy CT images using the radiological criteria described in the previous chapter, that is:

- RECIST 1.1 (Response Evaluation Criteria In Solid Tumours)

- Choi 
- MASS (Morphology, Attenuation, Size, and Structure)

- $\quad$ SACT (Size and Attenuation CT)

\section{Statistical Analysis}

Data were processed and analyzed by using Microsoft Excel 2011 Program. Sensitivity (Se), Specificity (Sp), Positive Predictive Value (PPV) and Negative Predictive value (NPV) were analyzed for each response criteria considering as gold standard a Progression Free Survival (PFS) of 250 days after the initiation of the therapy. Progression Free Survival is defined as that length of time of the antiangiogenic treatment until his suspension due to the progression of the metastatic disease ${ }^{[12]}$. The outcome was defined by the response to the therapy in terms of PFS: good clinical outcome if the PFS exceeding 250 days and negative clinical outcome if PFS were below 250 days. For statistical analysis we considered partial response (PR) and stable disease (SD) together because in advanced stage tumors the therapy can be considered effective not only in case of tumor regression but also in case of stable disease. In both cases the therapy can be continued, while the therapy had to be interrupted in cases of disease progression. For each criteria the major axis measurements of target lesions, which were at least $1 \mathrm{~cm}$, were being made at the level of the chest, abdomen and pelvis through the CECT. Non-over 10 target lesions for each patient were selected with an upper limit of 5 lesions per organ; in cases where an organ contained numerous lesions, the same targets were selected based on their preponderance.

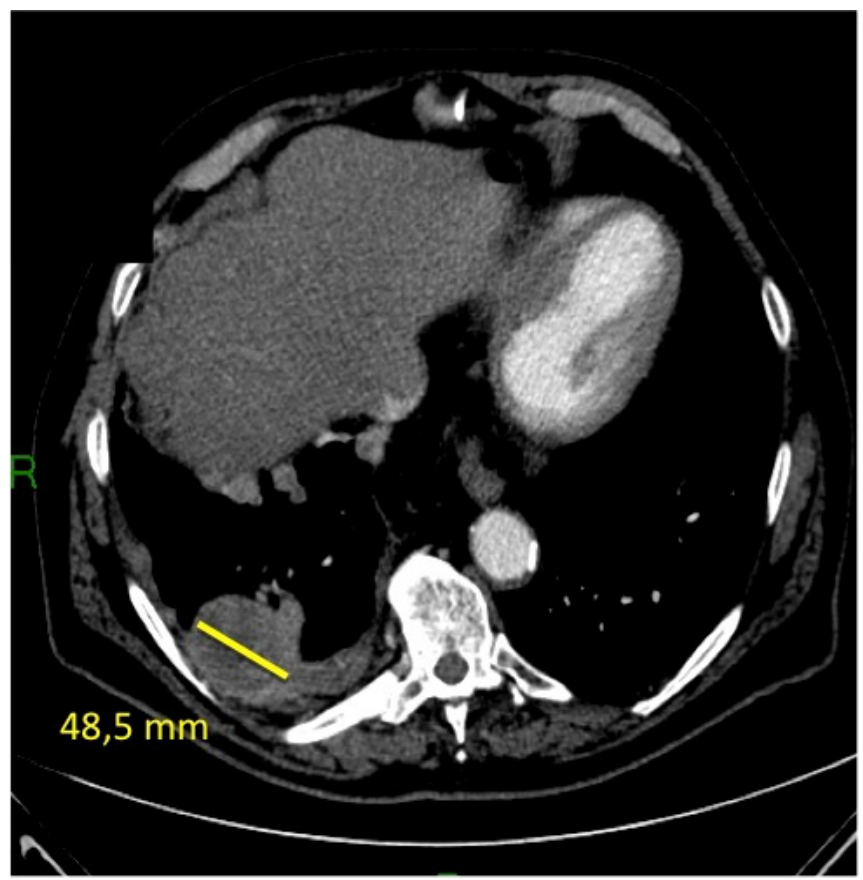

\section{Results}

The results are presented in table 1 and 2. Out of the 44 patients included in our study, 21 had a good clinical outcome, therefore a Progression Free Survival higher than 250 days while the remaining 23 patients had a Progression Free Survival below 250 days. In the group of 21 patients with PFS higher than 250 days, all radiological criteria had high predictive value, with a slightly higher sensitivity to RECIST and Choi criteria; as a matter of fact, both have identified 21 patients $(100 \%)$ as having a partial response, while MASS and SACT criteria identified 20 patients $(95.23 \%)$ as having a favorable response (Figure 1, Table 5). On the other hand, in the Group of 23 patients with negative outcome, therefore with PFS below 250 days, RECIST and Choi criteria have identified respectively 9 and 10 as patients with stable disease, while MASS and SACT criteria have identified respectively 11 and 10 patients as having an indeterminate response (Figure 2, Table 6). However, in the group of patients with a PFS exceeding 250 days, if we consider only partial or favorable response, RECIST criteria had the worst results, especially in the sensitivity area; in fact, among patients with a good clinical outcome, RECIST criteria have identified only 5 out of 21 patients $(23,80 \%)$ as having a partial response to the treatment, while the attenuation-based criteria, such as MASS, SACT and Choi criteria, identified respectively 15 (71.42\%), 14 (66.66\%) and $15(71.42 \%)$ patients as having a favorable response to the antiangiogenic treatment (Figure 3, Table 7).Those results demonstrated that the functional criteria based on attenuation (Choi, MASS and SACT) manage to identify more patients as "responder" compared to RECIST which instead consider them as stable.

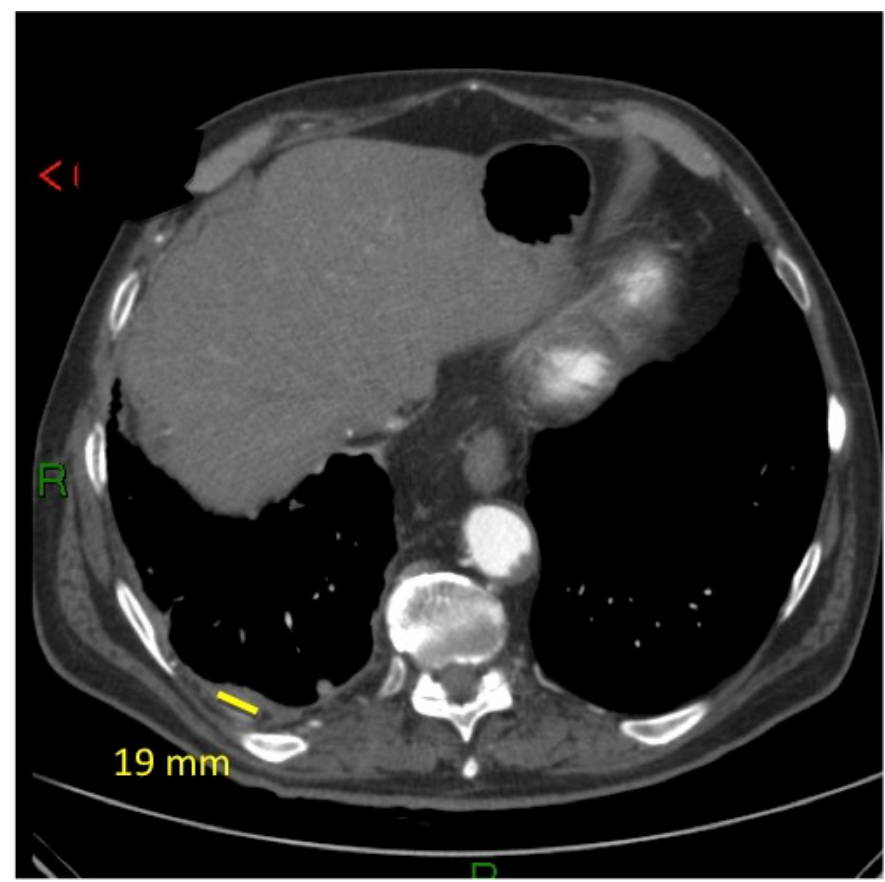

Figure1. Example of Partial or Favorable Response. Pleural lesion that presents a decrease in tumor size $>20 \%$ after treatment. All analyzed criteria classified it as Partial or Favorable Response. 


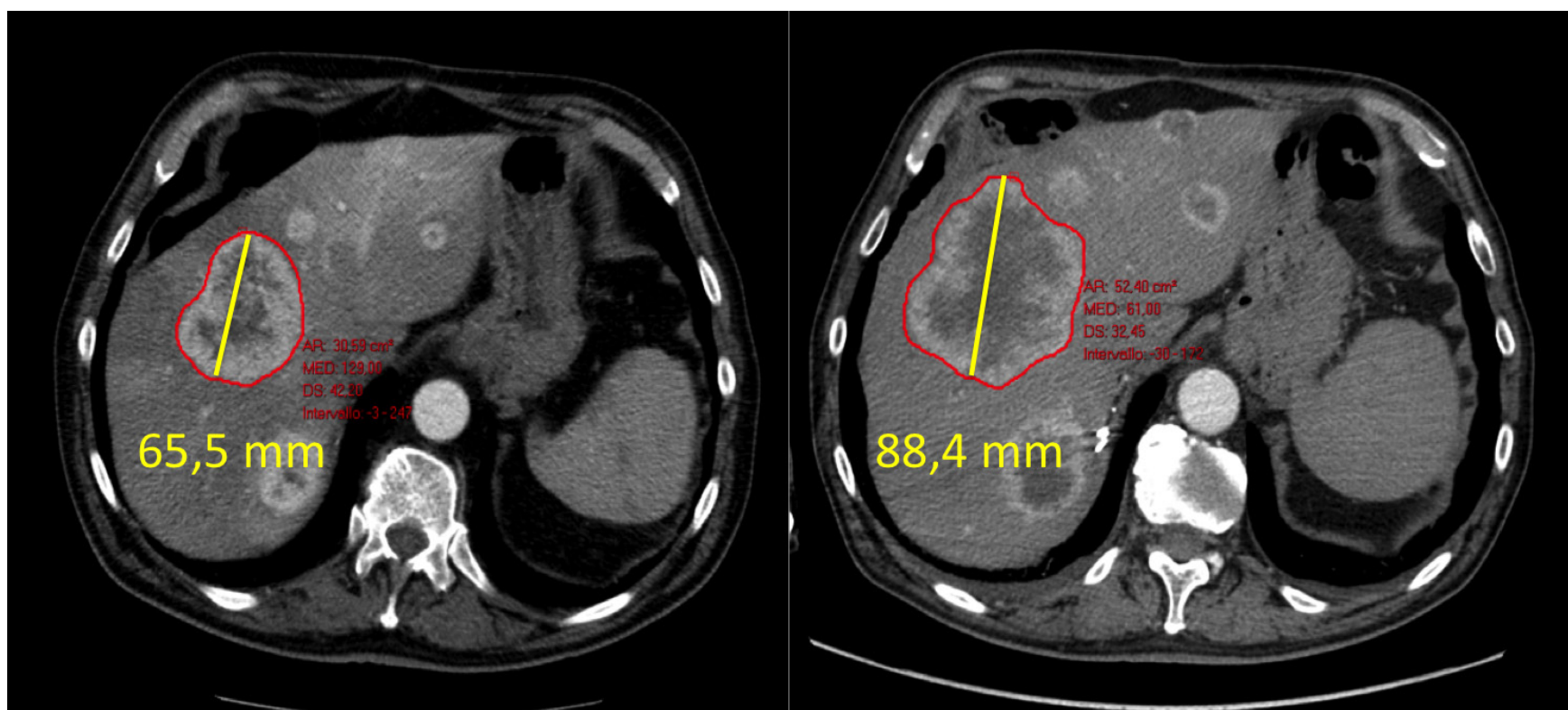

Figure 2. Example of Progressive Disease. Liver lesion considered as Progressive Disease according RECIST criteria due the increase in tumor size over 30\%; though, considered as Favorable/partial Response according MASS criteria due the central marked necrosis, according SACT criteria due the attenuation decrease over $40 \mathrm{HU}$ and according to $\mathrm{CHOI}$ criteria due the $15 \%$ decrease of the enhancement.

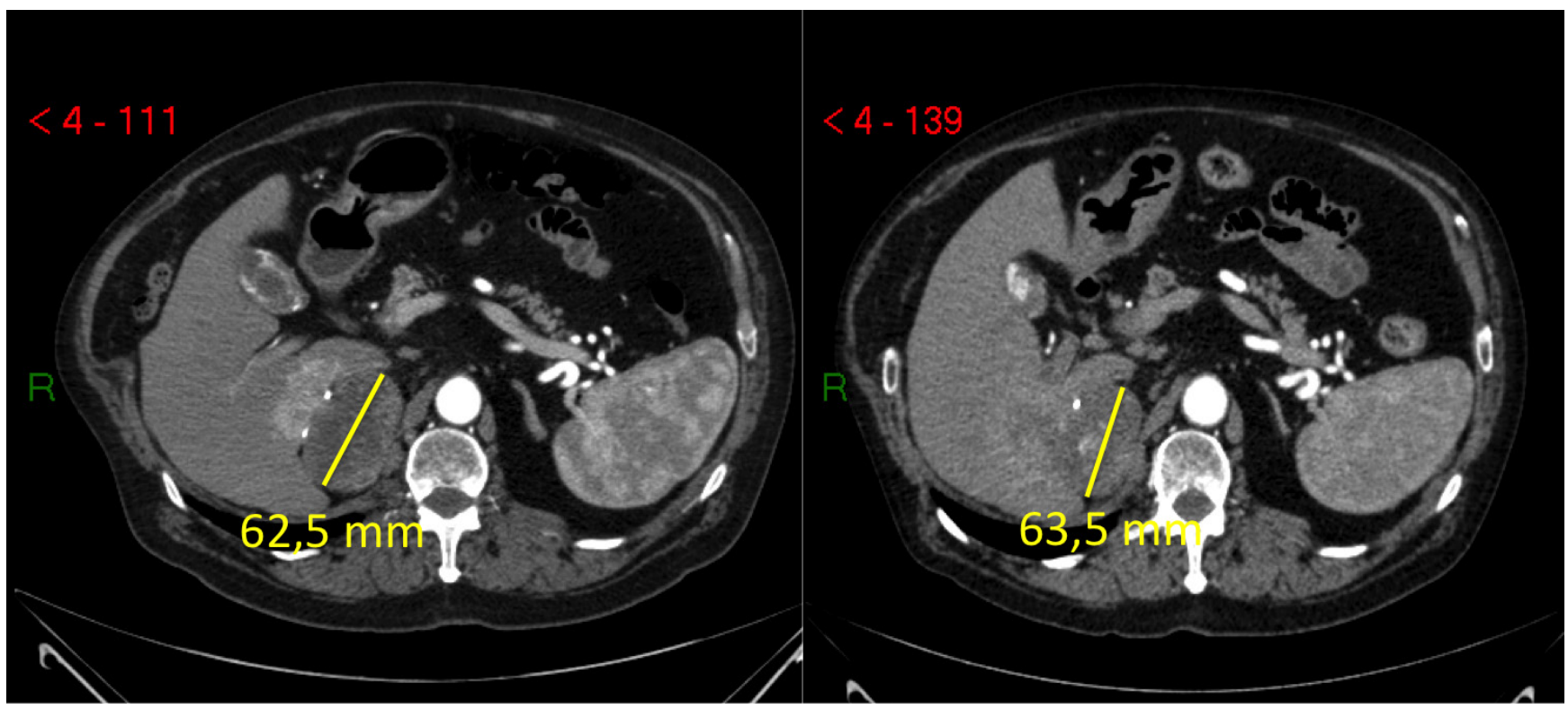

Figure 3. Example of Stable Disease for all the criteria. The lesion has not changed in size or attenuation.

Table 5. Sensitivity, specificity and accuracy in patients with PFS $>250$ days

\begin{tabular}{|c|c|c|c|}
\hline & Sensitivity & Specificity & Accuracy \\
\hline RECIST & $100 \%$ & $57,14 \%$ & $68,18 \%$ \\
\hline Choi & $100 \%$ & $52,38 \%$ & $70,45 \%$ \\
\hline MASS & $95,23 \%$ & $47,61 \%$ & $70,45 \%$ \\
\hline SACT & $95,23 \%$ & $52,38 \%$ & $68,18 \%$ \\
\hline
\end{tabular}

Table 6. Sensitivity, specificity and accuracy in patients with PFS $<250$ days

\begin{tabular}{|c|c|c|c|}
\hline & Sensitivity & Specificity & Accuracy \\
\hline RECIST & $39,13 \%$ & $60,86 \%$ & $70 \%$ \\
\hline Choi & $43,47 \%$ & $56,52 \%$ & $77,27 \%$ \\
\hline MASS & $47,82 \%$ & $52,17 \%$ & $77,27 \%$ \\
\hline SACT & $43,47 \%$ & $56,52 \%$ & $75 \%$ \\
\hline
\end{tabular}


Table 7. Sensitivity of radiological criteria, specificity and accuracy in patients with PFS $>250$ days considering Partial Response and Favorable Response only

\begin{tabular}{|c|c|c|c|}
\hline & Sensitivity & Specificity & Accuracy \\
\hline RECIST & $23,8 \%$ & $25 \%$ & $20,45 \%$ \\
\hline Choi & $71,42 \%$ & $38,09 \%$ & $52,27 \%$ \\
\hline MASS & $71,42 \%$ & $33,33 \%$ & $50 \%$ \\
\hline SACT & $66,66 \%$ & $33,33 \%$ & $47,72 \%$ \\
\hline
\end{tabular}

\section{Discussion}

Studying carefully the structural variations of the primary tumor and secondary lesions is important for evaluating the activity of antineoplastic therapies. The introduction of targeted antiangiogenic therapy led to a marked improvement in terms of both survival and in terms of Progression Free Survival (PFS) in patients with metastatic RCC. These progresses have led to a continuous evolution and improvement of the radiological criteria being able to identify the tumor behavior during therapy with tyrosine kinase inhibitors; in our case, Sunitinib and Sorafenib. In our study, in the comparison of radiological criteria in antiangiogenic therapy of metastatic RCC, using dimensional measurement methods and attenuation on CECT, for each criterion we got comparable predictive values. However, in a more accurate analysis, it appear that despite all the radiological criteria studied had a high predictive value in identifying patients with PFS exceeding 250 days, RECIST criteria have a slightly higher sensitivity; these in fact are the best in distinguish between patients who can continue the therapy from patients who must suspend it due to the progression of the disease. Our study shows that, among patients with a PFS exceeding 250 days, attenuation-based criteria (MASS, SACT and Choi) identify a greater number of "responder" compared to RECIST. Dimensional criteria (RECIST), in fact, in the context of patients that can continue the therapy, consider cases with stable disease in greater number than cases with partial/favorable response. Conclusively, within three months from the initiation of therapy, the partial/favorable sensitivity of attenuation-based criteria is higher than the partial response sensitivity according RECIST 1.1 criteria.

\section{Comments}

Background Usually for the evaluation of therapy response we use RECIST 1.1 that has been based on changes in target lesion sizes and the presence of new metastases. However, different studies underlined the inadequacy of Response Evaluation Criteria in Solid Tumors (RECIST) in assessing therapeutic response in patients treated with antiangiogenic targeted therapy. For this reason criteria that evaluate attenuation were purposed and for example CHOI had been accepted for GIST treated with TK-inibitor like sunitinib. For the evaluation of Renal metastatic clear cell cancer recent data show that objective analysis of both tumor size and attenuation on CECT improves response evaluation. In particular, decrease in tumor size and decrease in attenuation on CECT more frequently correlate better with increased progression-free survival than lesion size changes only. For this reason two new criteria were proposed: MASS: Mass, Attenuation, Size and Structure (MASS) - SACT: Size and Attenuation CT) criteria. These criteria for imaging response categories are using favorable, indeterminate and unfavorable response and besides changes in size and new lesions take into consideration the central necrosis and the decrease in attenuation or new enhancement and the central fill-in.

\section{REFERENCES}

[1] Siegel R, Naishadham D, Jemal A. Cancer statistics. CA Cancer J Clin 2013; 63: 11-30

[2] Ridge CA, Pua BB, Madoff DC. Epidemiology and staging of renal cell carcinoma. Semin Intervent Radiol 2014; 31(1): 3-8

[3] Wong-Ho C, Linda MD, Susan SD, Epidemiology and risk factors for kidney cancer. Nat Rev Urol 2010; 7 (5): 245-257

[4] Rouviere O, Bouvier R, Negrier S, Badet L, Lyonnet D. Non metastatic renal cell carcinoma: is it really possible to define rational guidelines for post treatment follow up. Nat Clin Pract Oncol 2006; 3: 200-213

[5] Sugimura K, Ikemoto SI, Kawashima H, Nishisaka N, Kishimoto T. Microscopic hematuria as a screening marker for urinary tract malignancies. Int J Urol 2001; 8(1): 1-5

[6] De Kernion JB, Lowitz B, Casciato D. Urinary tract cancers. In: Casciato D and Lowitz B. Manual of Clinical Oncology, Boston: Little, Brown and Company, 1998; 198-219

[7] Eisenhauer EA, Therasse P, Bogaerts J, et al. New response evaluation criteria in solid tumors: recise RECIST guideline (version 1.1). Eur J Cancer 2009. 228-247.

[8] Choi H. Charnsangavej C, Faria SC, Macapinlac HA, Burgess MA, Patel SR, Chen LL, Podoloff DA, Benjamin RS. Correlation of Computed Tomography and Positron Emission Tomography in patients with metastatic gastrointestinal tumor treated at a single institution with imatinib mesylate: Proposal of new computed tomography response criteria. $J$ Clin Oncol 2007; 25:1753-1759

[9] Choi H. Response evaluation of gastrointestinal stromal tumors. The oncologist 2008, 13 (suppl 2); 4-7.

[10] Smith AD, Shah SN, Rini B, Lieber ML, Remer EM. Morphology, attenuation, size and structure (MASS) Criteria: Assessing response and predicting clinical outcome in metastatic renal cell carcinoma on antiangiogenetic targeted therapy. AJR Am J Roentgenol 2010 Jun; 194(6):1470-8

[11] Smith AD, Lieber ML, Shah SN. Assessing tumor response and detenting recurrence in metastatic renal cell carcinoma on targeted therapy: importance of size and attenuation on contrast-enhanced CT. AJR Am J Roentgenol 2010 Jan; 194(1):157-165

[12] Booth CM, Eisenhauer EA. Progression-free survival: meaningful or simply measurable? JCO 2012 April; 30 (10): 1030-1033. 